\title{
CLINICAL CHARACTERISTICS, PROGNOSIS, AND TREATMENT OF PELVIC CRYPTORCHID SEMINOMA
}

\author{
Ye-Xiong Li, M.D., * Philippe A. Coucke, M.D., ${ }^{\dagger}$ Tu-Nan Qian, M.D., ${ }^{*}$ Yi-Rong Huang, M.D., ${ }^{*}$ \\ Da-Zhong Gu, M.D., ${ }^{*}$ René-Olivier Mirimanoff, M.D. ${ }^{\dagger}$ and Zi-Hao Yu, M.D.*
}

*Department of Radiation Oncology, Cancer Hospital and Institute, Chinese Academy of Medical Sciences, Beijing, P.R. China; and 'Department of Radiation Oncology, Centre Hospitalier Universitaire Vaudois (CHUV), Lausanne, Switzerland

\begin{abstract}
Purpose: To analyze the clinical characteristics, prognosis, and treatment outcome of pelvic cryptorchid seminoma

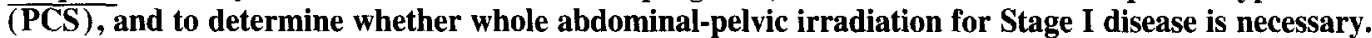

Methods and Materials: From 1958 to 1991, 60 patients with PCS were treated at the Cancer Hospital of Chinese Academy of Medical Sciences, Beijing. They presented with a lower abdominal mass and showed a predominance for the right side. A high proportion of patients with PCS [26 of $60(43 \%)$ ] had metastatic disease, compared to $20 \%$ of those with scrotal scminoma, and there was a tendency toward a higher frequency of pelvic nodal metastases. There were 34 Stage I, 6 Stage IIA, 11 Stage IIB, 5 Stage III, and 4 Stage IV patients. Of these 60 patients, 56 underwent laparotomy with or without cryptorchiectomy ( 37 radical orchiectomy, 7 partial orchiectomy, and 12 biopsy of the primary or cervical node), and 4 cervical node biopsy only. All patients were further treated with radiotherapy, chemotherapy, or a combination of both. Patients with Stage I and II disease received radiotherapy, whereas patients with Stage III and IV were treated with chemotherapy.

Results: The overall and disease-free survivals at 5 and 10 years were $92 \%$ and $87 \%$, and $88 \%$ and $84 \%$, respectively. The 5- and 10-year survivals were $100 \%$ for Stage I, $94 \%$ and $87 \%$ for Stage II, and $56 \%$ and $42 \%$ for Stage III/IV, respectively $(p<0.05)$. Volume of irradiation, i.e., whole abdominal-pelvic radiotherapy ( 10 patients), versus hockey-stick encompassing paraaortic, ipsilateral iliac nodes and the primary tumor or tumor bed (17) did not influence outcome in Stage I patients. Five patients relapsed within 2-12 ycars after treatment and four of these patients were successfully salvaged. Four patients developed a second malignant tumor and died.

Conclusion: Stage I and II PCS can be adequately controlled by radiotherapy regardless of the surgical procedure. Whole abdominal-pelvic irradiation for Stage I and IIA disease is not required, and fields can be limited to the paraaortic, ipsilateral iliac nodes and primary tumor or tumor bed. We recommend platinum-based chemotherapy for Stage IIB -IV PCS. (C) 1997 Elsevier Science Inc.
\end{abstract}

Seminoma, Cryptorchidism, Radiotherapy.

\section{INTRODUCTION}

The incidence of tumors arising in cryptorchid testes is $15-$ 45 times higher than that occurring in a scrotal testis $(4,8$, $14,18,24,34)$. Moreover, in undescended testes located in the abdomen which comprise $15 \%$ of cryptorchid testes, the risk of tumor development is even six times higher than for inguinal location (23). Most of those tumors arising in ectopic testes are pure seminoma $(1,14)$. However, in comparison to scrotal seminoma, they show a tendency to a higher frequency of nodal metastases $(11,19)$.

The prognosis for seminoma arising in cryptorchid testes is still a matter of debate. Early clinical study suggests a worse prognosis compared to scrotal seminoma. However, a number of recent series report an excellent outcome when patients receive appropriate treatment $(11,20,29)$.
In contrast to postorchiectomy radiation to paraaortic and ipsilateral pelvic nodes, which is considered the standard treatment for scrotal pure seminomas $(2,5,6,12$. 35 ), there is a controversy concerning the adequate volumes to be treated in the case of Stage I pelvic cryptorchid seminoma (PCS) (11). However, owing to the rarity of the disease and the variety of treatment options, definite conclusions cannot be drawn $(11,20,29)$. The aim of this retrospective review was to define overall prognosis and to assess if whole abdominal-pelvic radiotherapy is required for the treatment of Stage I PCS.

\section{METHODS AND MATERIALS}

Between June 1958 and December 1991, 373 patients were treated for a testicular seminoma at the Cancer Hos-
Reprint requests to: Ye-Xiong Li, M.D., Department of Radiation Oncology, Centre Hospitalier Universitaire Vaudois
(CHUV), 1011-Lausanne, Switzerland.

Accepted for publication 29 November 1996. 
Table 1. Stage for testicular seminoma

\begin{tabular}{ll} 
Stage & Definitions \\
\hline I & $\begin{array}{c}\text { Primary tumor confined to the testis or extending to } \\
\text { adjacent tissue or organ }\end{array}$ \\
II & $\begin{array}{l}\text { Infradiaphragmatic node metastases } \\
<5 \mathrm{~cm} \text { in diameter }\end{array}$ \\
IIA & $\begin{array}{l}<\mathrm{cm} \text { in diameter } \\
\text { IIB }\end{array}$ \\
III & Supradiaphragmatic node metastases \\
IV & Extralymphatic metastases \\
\hline
\end{tabular}

pital, Chinese Academy of Medical Sciences (CAMS), Beijing, China. The diagnosis of pure seminoma was confirmed in all cases by histopathologic review. Of these patients, 136 had a history of cryptorchidism, but 25 of them had undergone prior orchiopexy ( 23 inguinal and 2 pelvic cryptorchidism). From the remaining 111 patients, there were 43 inguinal localizations and 68 presented with a pelvic form of cryptorchidism. Of these 68 patients with PCS, 60 patients were primarily treated at our hospital and 8 patients were referred for recurrence after previous treatment elsewhere. The former 60 patients form the basis of this retrospective review. The characteristics and outcome of these 60 patients were compared to those observed in the 237 patients with seminoma arising in normally descended testes (scrotal seminoma).

All patients were staged according to the system described in Table 1. Patient workup included medical history, physical examination, blood chemistry, and chest X ray. Radiological examinations for infradiaphragmatic disease was done in 45 patients. Intravenous pyelography was performed in 18 patients, bipedal lymphangiography in 3, abdominal-pelvic computed tomography and/or ultrasound in 28 , and renogram in 30 . Tumor markers included alphafetoprotein (AFP), and $\beta$-human chorionic gonadotrophin $(\beta \mathrm{HCG})$ in 20 . Staging laparotomy with careful examination of abdominal and pelvic nodes was done in 25 patients.

\section{Treatment}

Surgery. All patients with PCS underwent laparotomy, except for four patients who had cervical node biopsy. The technique and extent of surgery for the primary was decided individually according to the tumor extension and presence of adhesions. Of the 56 patients with laparotomy, 37 had complete orchiectomy, 17 had partial orchiectomy or biopsy of the primary tumor, and 2 underwent cervical node biopsy. In Table 2, the extent of the resection, partial or complete, refers to the type of surgery for the primary tumor. The lymph nodes were not resected except for two Stage I patients who had retroperitoneal node dissection. The rate of complete resection of the primary tumor decreased with incrcasing stage: $79 \%$ for Stage I, $83 \%$ for Stage IIA, $36 \%$ for Stage IIB, and $11 \%$ for Stages III and IV.

Radiotherapy. The treatment option was dependent on the stage, size, and extent of nodal metastases. Patients
Table 2. Surgical approaches for primary tumor according to stage

\begin{tabular}{|c|c|c|c|c|c|c|}
\hline & \multicolumn{6}{|c|}{ Stage } \\
\hline & I & IIA & IIB & III & IV & Total \\
\hline \multicolumn{7}{|l|}{ Laparotomy combined with } \\
\hline Radical cryptorchicctomy & 27 & 5 & 4 & 0 & 1 & 37 \\
\hline Partial cryptorchiectomy & 3 & 0 & 2 & 1 & 1 & 7 \\
\hline Biopsy of the primary & 4 & 1 & 4 & 1 & 0 & 10 \\
\hline $\begin{array}{l}\text { Cervical node biopsy } \\
\text { only }\end{array}$ & 0 & 0 & 0 & 2 & 0 & 2 \\
\hline No laparotomy & & & & & & \\
\hline $\begin{array}{l}\text { Cervical node biopsy } \\
\text { only }\end{array}$ & 0 & 0 & $1^{*}$ & 1 & 2 & 4 \\
\hline
\end{tabular}

* This patient had no histologic confirmation prior to therapy and developed cervical node metastases during chemothcrapy.

with Stage I and II diseases were usually treated with radical radiotherapy with or without consolidation chemotherapy, whereas in patients with Stage III and IV the treatment choice was chemotherapy with consolidation radiotherapy. Fifty-two of 60 patients were treated with radiotherapy, and in 30 of them this was the sole treatment after surgery. In 22 patients radiotherapy was combined with chemotherapy. Only eight patients reccived chcmotherapy alone after the initial diagnosis and/or surgical management (Table 3 ).

Before 1982, the patients were treated with a cobalt 60 unit, and thereafter, a linear accelerator ( $8 \mathrm{MV}$ ) was used. The volume was irradiated by parallel opposed anteriorposterior and posterior-anterior fields. The daily dose per fraction varied from 1 to $1.8 \mathrm{~Gy}$. The primary tumor site and areas at risk were approached by either whole abdominal-pelvic irradiation (WAPI) or a hockey-stick technique encompassing paraaortic, ipsilateral iliac nodes and the primary tumor or tumor bed. In case of WAPI, the whole abdominal-pelvic dose was raised to 15-20 Gy. However, liver and spleen were excluded from the start of WAPI, whereas kidneys received up to 15-20 Gy. At this dose level, a standard hockey stick technique replaced the WAPI. Forty-four of 51 patients with Stages I and II received radiotherapy with or without chemotherapy. Table 4 summarizes the treatment volumes and specifies the addition or not of chemotherapy to these patients. Of five

Table 3. Treatment by stage

\begin{tabular}{lrrrrrrr}
\hline & \multicolumn{7}{c}{ Stage } \\
\cline { 2 - 7 } & I & IIA & IIB & III & IV & Total \\
\hline RT alone & 21 & 1 & 6 & 2 & 0 & 30 \\
Chemo alone & 5 & 1 & 1 & 0 & 1 & 8 \\
RT + Chemo & 4 & 0 & 0 & 0 & 0 & 4 \\
Chemo + RT & 4 & 4 & 4 & 3 & 3 & 18 \\
\hline
\end{tabular}

$\mathrm{RT}=$ Radiotherapy; Chemo $=$ chemotherapy. 
Table 4. Radiation target volume for Stage I and II patients with or without chemotherapy

\begin{tabular}{lcccccc} 
& \multicolumn{2}{c}{ Hockey stick } & & \multicolumn{2}{c}{ WAPI } & \\
Stage & Alone & Chemo & & Alone & Chemo & $\begin{array}{c}\text { Local RT } \\
\text { plus chemo }\end{array}$ \\
\hline I & 11 & 6 & & 10 & 0 & 2 \\
IIA & 1 & 3 & & 0 & 0 & 1 \\
IIB & 1 & 2 & 5 & 1 & 1 \\
$\quad$ Total & 13 & 11 & & 15 & 1 & 4 \\
\hline
\end{tabular}

RT $=$ Radiotherapy Chemo $=$ chemotherapy; WAPI $=$ whole abdominal-pelvic irradiation.

patients with Stage III, two patients were treated with radiotherapy alone and three with combination of chemotherapy and radiotherapy. Three patients with Stage IV disease received radiotherapy to residual tumor following chemotherapy.

In the early years of this study, prophylactic mediastinal and left supraclavicular irradiation was given to 2 of 34 Stage I and 3 of 17 Stage II patients.

The total dose to the target volume ranged from 20 to 40 Gy (median 30 ) for Stage I. In Stage II disease, the intradiaphragmatic dose was usually raised to $35 \mathrm{~Gy}$. After orchiectomy, a dose of 30-40 Gy was applied to the tumor bed. For those patients considered unresectable, the total dose to the primary tumor reached $40-60 \mathrm{~Gy}$.

Chemotherapy. Thirty of 60 patients were treated with chemotherapy. Eight of them received chemotherapy alonc and 18 received a combined treatment of chemotherapy followed by radiotherapy. Only in four patients was chemotherapy given as consolidation treatment after radiotherapy (Table 3 ). During the earlier years of this study, $\mathrm{N}$-formylsarcolycin, a nitrogen mustard analogue, was used alone (22) or combined with cyclophosphamide or vincristine (5). From 1985 on, more effective chemotherapy, i.e., platinum-based regimens, were used in three patients.

\section{Follow'-up and statistics}

Patients were followed up every 3 months for 1 year and thereafter every 6 months or once yearly. At each follow-up, they underwent a physical examination, complete blood count, serum biochemistry, and chest X rays. Since 1982, abdominal-pelvic CT scan and/or ultrasound were added to the routine follow-up procedures. Tumor markers, i.e., $\beta \mathrm{HCG}$ and AFP, were not routinely repeated. The median follow-up time was 18 years ( range 3-36).

The overall survival (OS) was calculated from the date of surgery. Disease-free survival (DFS) was calculated from the date of surgery until evidence of recurrence or progression. Updating of the medical files was done in December 1994, and survival data were calculated according to these. Survival curves were calculated according to the Kaplan-Meier method and differences between survival curves were analyzed with the log rank test. $\mathrm{Nu}$ merical data were compared with Student's $t$-test and comparison of table was done by chi-square analysis.

\section{RESULTS}

\section{Patient characteristics}

The median age of PCS patients was 38 years (range 17-70), whereas for those with scrotal seminoma (control group) it was 34 years (range 21-82). The peak age for PCS was between 30 and 50 years. The most frequently presenting symptoms were low abdominal mass and mass effect. The right to left ratio was 2:1. Thirteen patients had a contralateral cryptorchidism. One patient had synchronous contralateral scrotal seminoma (Table 5).

The size of the primary tumor ranged from 5 to $28 \mathrm{~cm}$, with a median size of $10 \mathrm{~cm}$. There was no correlation between stage and size: The median maximum diameter for Stages I, II, and III was $10 \mathrm{~cm}$, and for Stage IV it was $11 \mathrm{~cm}$. In contrast, scrotal seminoma had a median diameter of $6 \mathrm{~cm}$ ( range 2-15), significantly smaller than those in PCS. Eighteen of $60(30 \%)$ patients had invasion of the primary tumor into neighboring structures, especially the bowel ( 11 of 18 ), the bladder ( 8 of 18$)$, and the iliac vasculature ( 5 of 18 ). In $67 \%$ (12 of 18 ) of patients who underwent intravenous pyelography, we observed an incomplete (10) or complete (2) ureteral obstruction with hydronephrosis. The kidney function returned to normal levels after treatment in all patients.

Forty-three percent ( 26 of 60 ) of patients with PCS had metastatic disease to the lymph nodes, whereas only $20 \%$ ( 48 of 237) of patients from control group presented with

Table 5. Clinical characteristics of pelvic cryptorchid seminoma

\begin{tabular}{lc}
\hline \multicolumn{1}{c}{ Characteristics } & No. of patients \\
\hline Location & \\
Right & 41 \\
Left & 19 \\
Stage & 34 \\
I & 6 \\
IIA & 11 \\
IIB & 5 \\
III & 4 \\
IV & \\
Presenting symptom & 49 \\
Lower abdominal mass & 10 \\
Painful mass & 1 \\
Acute pain & \\
Other symptom & 5 \\
Leg edema & 1 \\
Urinary frequency & 1 \\
Urodynia & 1 \\
Difficult urination & \\
Contralateral cryptorchidism & 9 \\
Pelvic & 4 \\
Inguinal & \\
\hline
\end{tabular}


Table 6. Stage distribution in patients with pelvic cryptorchid seminoma compared to that in patients with scrotal seminoma

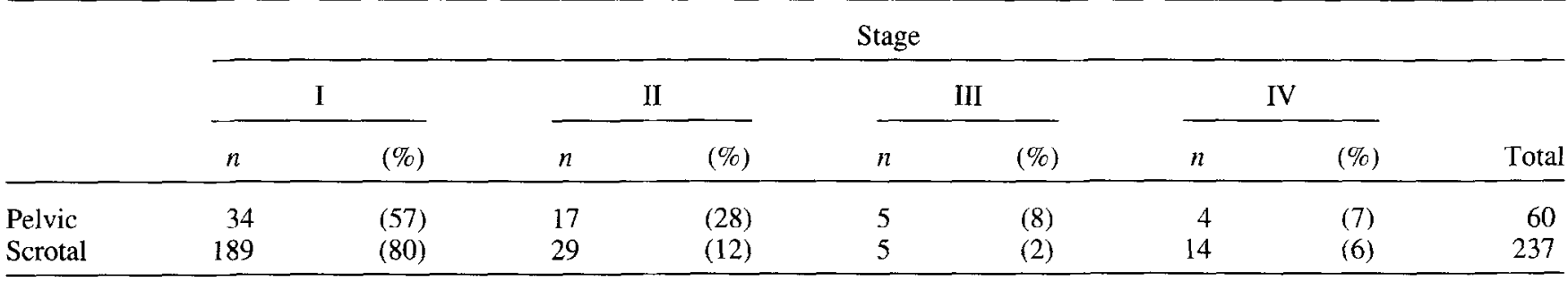

nodal disease $(p<0.01)$. Table 6 summarizes the differences in stage distribution between PCS and the control group. The anatomical distribution of nodal and/or distant metastases for PCS is shown in Table 7. Pelvic node involvement was unilateral except for one patient. Only one patient had pelvic node involvement without paraaortic node involvement. Pelvic nodal invasion in case of nodal metastasis was encountered in 23\% (6 of 26) of patients with PCS, whereas only $13 \%(6 / 48)$ of the control group had pelvic nodal involvement. However, this difference was not significant ( $p=0.23$ ).

No patient with PCS had inguinal node metastasis, except for one patient who had pathologically confirmed right-sided inguinal node metastases. The latter patient presented with left-sided PCS and synchronous rightsided scrotal seminoma. He had a bulky primary tumor in the pelvis and had no prior inguinal or scrotal surgery. At staging laparotomy, there were neither paraaortic nor pelvic nodes metastases.

\section{Survival}

The overall and disease-free survival rates at 5 and 10 years were $92 \%$ and $87 \%$, and $88 \%$ and $84 \%$, respectively. The overall 5-year and 10-year survival rates were $100 \%$ and $100 \%$ for Stage I, 94\% and $87 \%$ for Stage II, and $56 \%$ and $42 \%$ for Stage III/IV, respectively (Fig. 1). The differences in survival between Stages I, II, and III/IV were

Table 7. Anatomical distribution of nodal and distant metastases in patients with pelvic cryptorchid seminoma

\begin{tabular}{lc}
\hline \multicolumn{1}{c}{ Sites } & $n$ \\
\hline Stage IIA & 6 \\
$\quad$ Paraaortic only & \\
Stage IIB & 8 \\
$\quad$ Paraaortic only & 1 \\
Pelvic only & 2 \\
Paraaortic and pelvic & 2 \\
Stage III & 2 \\
$\quad$ Paraaortic and mediastinal & 1 \\
Paraaortic, mediastinal, and supraclavicular & 1 \\
Paraaortic, pelvic, mediastinal, and supraclavicular & 1 \\
Stage IV & 1 \\
Paraaortic, mediastinal, supraclavicular, and liver & 1 \\
Paraaortic, mediastinal, liver, kidney, and lung & \\
Paraaortic, pelvic, and liver & \\
Paraaortic, pelvic, mediastinal, supraclavicular, and lung & 1 \\
\hline
\end{tabular}

statistically significant $(p<0.05)$. For 17 Stage II patients, all 6 Stage IIA patients were alive without evidence of disease, whereas 2 of 11 Stage IIB patients died of disease. The 5-year and 10-year survival rates were $100 \%$ and $100 \%$ for Stage IIA, and $91 \%$ and $80 \%$ for Stage IIB, respectively. Owing to the small number of patients, there was no significant difference between Stages IIA and IIB.

Eleven patients died of other causes: four of a second malignant tumor, three of hypertension, two of cardiorespiratory disease, one of suicide, and one of unknown causes.

\section{Radiotherapy for Stages I and II}

To evaluate the potential impact of treated volume on outcome in Stage I PCS, we compared 17 patients treated prior to 1985 with a hockey-stick technique to 10 patients treated on whole ahdominal-pelvic irradiation (WAPI). None of these patients with WAPI received chemotherapy, whereas 6 of 17 patients with the hockey-stick technique received chemotherapy (Table 4 ). The remaining seven Stage I patients were not considered for the comparison between the hockey-stick technique and WAPI, because five of them received chemotherapy alone and two received local irradiation after chemotherapy. Our intent was basically to compare two modalities of extended field irradiation in Stage I disease. There was no difference between the two groups in locoregional recurrence whether DFS or OS is considered. Only one patient developed distant metastases after hockey-stick radiotherapy.

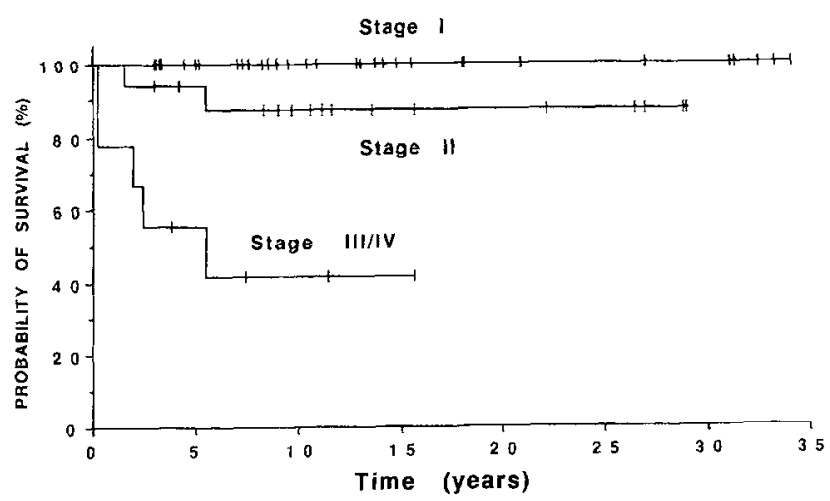

Fig. 1. Overall survival by stage in PCS: Stage I $(n=34)$; Stage II $(n=17)$; and Stage III/IV $(n=9)$. Log rank $p<0.05$. 
In Stage IIA, five of six patients received radiotherapy. Four of them were treated with the standard hockey-stick technique and only one was irradiated locally. One patient received chemotherapy alone. As mentioned in Tables 3 and 4 , chemotherapy was given prior to irradiation in all except one patient. All patients were alive and well. Of 11 Stage IIB patients, 6 were trealed with WAPI, 3 with the hockey-stick technique, 1 with local irradiation plus chemotherapy, and one with chemotherapy alone. In the subgroup of patients with Stage IIB who were treated with WAPI, there were no cancer-related events. Onc patient treated with hockey-stick-field therapy alone died from abdominal progressive discase.

\section{Recurrence}

Five of 60 patients ( $8 \%$ ) with PCS developed a relapse. The time to relapse ranged from 2 to 12 years, and in two patients, it occurred beyond 5 years.

Two Stage I patients developed relapse in infradiaphragmatic nodes after $\mathrm{N}$-formylsarcolycin, and one Stage I patient had liver relapse after WAPI. One Stage IIA patient, initially treated with $N$-formylsarcolycin, experienced supradiaphragmatic relapse. One patient with Stage III disease, who received radiotherapy alone, developed relapse in the abdomen. Four patients werc salvaged with radiotherapy or chemotherapy, and one died of disease.

\section{Complications}

One patient treated with orchiectomy, right hemicolectomy and radiotherapy reported chronic intermittent diarrhea, probably due to colectomy-induced malabsorption. Seven patients had bowel obstruction due to abdominal adhesion. Two of them occurred immediately after laparotomy but prior to radiation, and five occurred between 10 months and 21 years after treatment. Surgery was required to correct bowel obstruction in four cases.

Four patients developed a second malignant tumor (SMT) within 4-27 years after treatment of PCS. They died from scrotal seminoma, hepatic carcinoma, squamous cell carcinoma of the lung, and rectal adenocarcinoma. Three patients were initially treated with chemotherapy alone, and only one patient with apical lung cancer was previously treated with infradiaphragmatic radiation.

\section{DISCUSSION}

Seminoma arising in cryptorchid testes is rare, accounting for $1-7 \%$ of all seminomas $(4,11,20,22)$. PCS is even more exceptional, with only $5 \%$ seminomas arising in an abdominally located undescended testis (11). However, a higher incidence has been reported in China (29, 30 ). To our knowledge, this series represents the largest cohort of PCS ever reported. In our experience, seminoma in cryptorchid testis represents $30 \%$ of testicular seminoma, with $18 \%$ PCS and $12 \%$ inguinal cryptorchid seminoma.
In accordance with Gilliland and Key, there is a trend toward a higher incidence in the third decade (13). As observed by Willan and McGowan, there is a right predominance which seems also to be the case in scrotal seminoma (33). In contrast to scrotal scminoma, PCS tends to present initially with bulky primary disease, often invading or compressing surrounding organs. This results in a unique clinical presentation, characterized by leg edema, urinary frequency, and urodynia. Furthermore, PCS, by its location in the pelvis and retroperitoneum and its propensity for lymph node metastases, can impair kidney drainage more frequently than scrotal seminoma.

\section{Pathways of tumor spread}

Lymph node metastases are more frequent in PCS than in scrotal seminoma. An incidence of $30-76 \%$ has been reported in the literature $(11,27,29,30)$. In the present series, the incidence of $43 \%$ is significantly higher than the nodal involvement encountered in our control group of scrotal seminoma $(20 \%)$. This higher incidence of nodal involvement may be explained by a delay in diagnosis in PCS, compared to scrotal and/or inguinal seminoma.

The pattern of lymphatic spread is comparable to that of scrotal seminoma, with the major pathway being the paraaortic nodes. Furthermore, our data from PCS reveal that the iliac or paraaortic metastases may occur alone or in combination, regardless of the type of prior surgical intervention. The incidence of pelvic nodes is higher than for scrotal seminoma ( $23 \%$ vs. $13 \%$ ), and therefore, patients should be routinely investigated at the pelvic level. Inguinal nodal metastases are rare in PCS. Only one patient who presented with synchronous PCS and scrotal seminoma had inguinal nodes. In comparison, 4 of 237 patients with scrotal seminoma had inguinal disease. All five of these patients had bulky abdominal masses without prior inguinal or abdominal surgery. This potentially i1lustrates the disruption of the normal lymphatic pathways resulting in inguinal nodal involvement. Peritoneal metastases are rare, and in contrast to Gauwitz and Zagars, who found an $18 \%$ ( 3 of 17) incidence at presentation (11), only one patient in the present series had proven peritoneal implants at autopsy.

\section{Management and prognosis}

Surgery alone is not considered to be curative in PCS. The resection rate for primary tumor in the present series was $62 \%$ (37 of 60). Frequently resection is made difficult by dense fibrosis, abdominal adhesion, and local invasiveness of the tumor itself. Nevertheless, the regional control rate was $100 \%$, owing to the addition of radiotherapy and/or chemotherapy. Primary tumor burden and extent of residual tumors after surgery do not affect outcome; seminoma is highly sensitive to cytoreductive treatment. From our data, a complete resection of primary tumor is thus not a basic requirement for patients with PCS. 
Patients with Stage I PCS have an excellent prognosis after WAPI or hockey-stick radiation. Although nonrandomized, the comparison between patients treated with a hockey-stick technique and those treated with WAPI yields no significant difference in OS and DFS. Therefore, to reduce the potential risk of treatment-related late complication $(16,32)$, we recommend a hockey-stick technique encompassing paraaortic, ipsilateral iliac nodes and the primary tumor or tumor bed for Stage I and IIA PCS. It must be emphasized that these patients did not in fact receive whole abdominal-pelvic irradiation, since the liver and spleen were shielded from the start of treatment and at 15-20 Gy, WAPI was replaced by a hockey-stick technique. The differences in treatment volumes merely illustrate the lack of knowledge in biology and outcome in PCS. Furthermore, in contrast to Gauwitz and Zagars (11), we feel that whole pelvic irradiation is not required unless therc is bilateral inguinal, pelvic nodal involvement, or obvious peritoneal implants.

For patients with Stage IIB scrotal seminoma, the literature reports excellent survival figures, provided those patients are treated with whole abdominal irradiation (31, 33 ). It is interesting to note that five of the six patients with Stage IIB PCS treated with WAPI alone are alive with no evidence of disease (NED), whereas the single one treated on the paraaortic field only died from disease. However, in bulky seminoma, platinum-based chemotherapy is currently favored by most authors $(6,25,26)$.

Prophylactic mediastinal and/or supraclavicular irradiation is no longer required in patients with Stage II disease. None of the patients with infradiaphragmatic radiotherapy in this series developed a supradiaphragmatic relapse. This is in accordance with the recent therapeutic trends in bulky Stage II seminoma (7, 21, 25, 28, 33).

Survival for Stage III and IV PCS was poor, merely illustrating the lack of effective chemotherapy in earlier years. Current standard treatment for advanced stage seminoma includes platinum-based chemotherapy, with response rates reported to be around $85 \%(9,10,17$, 22,26 ).

The absence of in-field recurrence in Stage I PCS illustrates the fact that doses in the range of $30-35$ Gy are effective. As is the case for scrotal seminoma, smaller doses are probably able to control subclinical disease (12), but we do not have data to support this hypothesis. On the other hand, the local control rates obtained with $40-60$ Gy on bulky disease or residual primary suggests that $40 \mathrm{~Gy}$ is probably enough.

\section{Relapse/complications}

Two patients with PCS developed a relapse beyond 5 years, suggesting that late relapse is not a rare event. Therefore, patients with PCS should be followed up on a regular basis even after 5 years ( 3 ).

In this series, there were neither abdominal nor pelvic relapses in patients with Stage I and II PCS treated with radiotherapy. However, two of eight patients treated with $\mathrm{N}$-formylsarcolycin alone developed an abdominal or pelvic recurrence. More effective chemotherapy with platinum-based regimens might probably have prevented those relapses.

Abdominal complications, especially bowel obstruction are frequently encountered among patients with PCS. This can partly be explained by surgical intervention or location of the primary, which may result in abdominal adhesion.

\section{CONCLUSION}

The prognosis in PCS is excellent regardless of the extent of the primary surgery, provided the patients are treated with curative radiotherapy and/or chemotherapy. In case of unresectable primary, a high local control rate can be obtained with radiotherapy alone. For patients with Stage I and IIA disease, the absence of a significant survival benefit for WAPI argues in favor of the limitation of treatment volumes to paraaortic and ipsilateral iliac nodes including the primary tumor or tumor bed. For Stage IIB disease we would favor platinum-based chemotherapy, although in the present retrospective review, WAPI yielded excellent results. As is the case for Stage III and IV scrotal seminoma, platinum-based chemotherapy is the treatment of choice for advanced-stage PCS.

\section{REFERENCES}

1. Abratt, R. P.; Reddi, V. B.; Sarembock, L. A. Testicular cancer and cryptorchidism. Br. J. Urol. 70:656-659; 1992.

2. Babaian, R. J.; Zagars, G. K. Testicular seminoma: The MD Anderson experience. An analysis of pathological and patient characteristics and treatment recommendations. J. Urol. 139:311-314; 1988 .

3. Baniel, J.; Foster, R. S.; Gonin, R.; Messemer, J. E.; Donohue, J. P.; Einhorn, L. H. Late relapse of testicular cancer. J. Clin. Oncol. 13:1170-1176; 1995.

4. Batata, M. A.; Chu, F. C. H.; Hilaris, B. S.; Whitmore, W. F.; Golbey, R. B. Testicular cancer in cryptorchids. Cancer 49:1023-1080; 1982.

5. Bayens, Y. C.; Helle, P. A.; Van Putten, W. L. J.; Mali, S. P. M. Orchidectomy followed by radiotherapy in 176 stage I and II testicular seminoma patients: Benefits of a 10year follow-up study. Radiother. Oncol. 25:97-102; 1992.

6. Dosmann, M. A.; Zagars, G. K. Postorchiectomy radiotherapy for Stages I and II testicular seminoma. Int. J. Radiat. Oncol. Biol. Phys. 26:381-390; 1993.

7. Evensen, J. R.; Fossa, S. D.; Kjellevold, K.; Lien, H. H. Testicular seminoma: Analysis of treatment and failure for stage II disease. Radiother. Oncol. 4:55-61; 1985.

8. Farrer, J. H.; Walker, A. H.; Rajfer, J. Management of the postpubertal cryptorchid testis: A statistical review. J. Urol. 134:1071-1076; 1985.

9. Fossa, S. D.; Droz, J. P.; Stoter, G.; Kaye, S. B.; Vermeylen, K.; Sylvester, R. Cisplatin, vincristine and ifosphamide combination chemotherapy of metastatic sem- 
inoma: Results of EORTC trial 30874. Br. J. Cancer $71: 619-624 ; 1995$.

10. Friedman, E. L.; Garnick, M. B.; Stomper, P. C.; Mauch, P. M.; Harington, D. P.; Richie, J. P. Therapeutic guidelines and results in advanced seminoma. J. Clin. Oncol. 3:13251332; 1985.

11. Gauwitz, M. D.; Zagars, G. K. Treatment of seminoma arising in cryptorchid testes. Int. J. Radiat. Oncol. Biol. Phys. 24:153-159; 1992.

12. Giacchetti, S.; Raoul, Y.; Wibault, P.; Droz, J. P.; Court, B.; Eschwege, F. Treatment of Stage I testis seminoma by radiotherapy: Long-term results. A 30-year experience. Int. J. Radiat. Oncol. Biol. Phys. 27:3-9; 1993.

13. Gilliland, F. D.; Key, C. R. Male genital cancers. Cancer 75:295-315; 1995.

14. Halme, A.; Kellokumpu-Lehtinen, P.; Lehtonen, T.; Teppo, L. Morphology of testicular germ cell tumours in treated and untreated cryptorchidism. Br. J. Urol. 64:78-83; 1989.

15. Hamilton, C.; Horwich, A.; Easton, D.; Peckham, M. J. Radiotherapy for stage I seminoma testis: Results of treatment and complications. Radiother. Oncol. 6:115-120; 1986.

16. Hanks, G. E.; Peters, T.; Owen, J. Seminoma of the testis: Long term beneficial and deleterious results of radiation. Int. J. Radiat. Oncol. Biol. Phys. 24:913-919; 1992.

17. Horwich, A.; Dearnaley, D. P.; Duchesne, G. M.; Williams, M.; Brada, M.; Peckham, M. J. Simple nontoxic treatment of advanced metastatic seminoma with carboplatin. J. Clin. Oncol. 7:1150-1156; 1989.

18. Jones, B. J.; Thornhill, J. A.; O'Donnell, B.; Kelly, D. G.; Walsh, A.; Fennelly, J. J.; Fitzpatrick, J. M. Influence of prior orchiopexy on stage and prognosis of testicular cancer. Eur. Urol. 19:201-203; 1991.

19. Jonsson, K.; Wallace, S.; Jing, B. S.; Boyle, L. F.; Iohnson, D. E. Lymphangiography in patients with malignancy in a non-descended testicle. J. Urol. 119:614-617; 1978.

20. Kulkarni, J. N.; Kamat, M. R. Tumors in undescended testis. J. Surg. Oncol. 46:257-260; 1991 .

21. Laukkanen, E. L.; Olivotto, I.; Jackson, S. Management of seminoma with bulky abdominal disease. Int. J. Radiat. Oncol. Biol. Phys. 14:227-233; 1988.

22. Loehrer, P. J.; Birch, R.; Williams, S. D.; Greco, F. A.; Einhorn, L. H. Chemotherapy of metastatic seminoma: The
Southeastern Cancer Study Group experience. J. Clin. Oncol. 5:1212-1220; 1987.

23. Martin, D. C. Malignancy in the cryptorchid testis. Urol. Clin. North Am. 9:371-376; 1982.

24. Martin, D. C.; Menck, H. R. The undescended testis: Management after puberty. J. Urol. 114:77-79; 1975.

25. Mason, B. R.; Kearsley, J. H. Radiotherapy for stage 2 testicular seminoma: The prognostic influence of tumor bulk. J. Clin. Oncol. 6:1856-1862; 1988.

26. Mencel, P. J.; Motzer, R. J.; Mazumdar, M.: Vlamis, V.: Bajorin, D. F.; Bosl, G. J. Advanced seminoma: Treatment results, survival, and prognostic factors in 142 patients. J. Clin. Oncol. 12:120-126; 1994.

27. Raina, V.; Shukla, N. K.; Gupta, N. P.; Deo, S.; Rath, G. K. Germ cell tumours in uncorrected cryptorchid testis at Institute Rotary Cancer Hospital. New Delhi. Br. J. Cancer 71:380-382; 1995.

28. Sagerman, R. H.; Kotlove, D. J.; Regine, W. F.; Chung, C. T.; King, G. A. Stage II seminoma: Results of postorchiectomy irradiation. Radiology 172:565-568; 1989.

29. Sham, J. S. T.; Choy, D.; Chan, K. W.; Choi. P. H. K. Seminoma of normally-descended and cryptorchid testis. Eur. J. Surg. Oncol. 16:33-36; 1990.

30. Shi, X. H.; Liu, T. F. Radiotherapy for seminoma arising in undescended testis: Analysis of 103 cases. Tumor 7:113$115 ; 1987$ (in Chinese).

31. Smalley, S. R.; Evans, R. G.; Richardson, R. L.; Farrow, G. M.; Earle, J. M. Radiotherapy as initial treatment for bulky stage II testicular seminomas. J. Clin. Oncol. 3:1333$1338 ; 1985$.

32. Vallis, K. A.; Howard, G. C. W.; Duncan, W.; Cornbleet. M. A.; Kerr, G. R. Radiotherapy for stages I and II testicular seminoma: Results and morbidity in 238 patients. Br. J. Radiol. 68:400-405; 1995.

33. Willan, B. D.; McGowan, D. G. Seminoma of the testis: A 22-year experience with radiation therapy. Int. J. Radiat. Oncol. Biol. Phys. 11:1769-1775; 1985.

34. Wobbes, T.; Schraffordt, K. H.; Oldhoff, J. The relation between testicular tumours, undescended testes, and inguinal hernias. J. Surg. Oncol. 14:45-51; 1980.

35. Zagars, G. K.; Babaian, R. J. Stage I testicular seminoma: Rationale for postorchiectomy radiation therapy. Int. J. Radiat. Oncol. Biol. Phys. 13:155-162: 1987. 